\title{
Zukunftsfähigkeit der Datenschutz- Grundverordnung
}

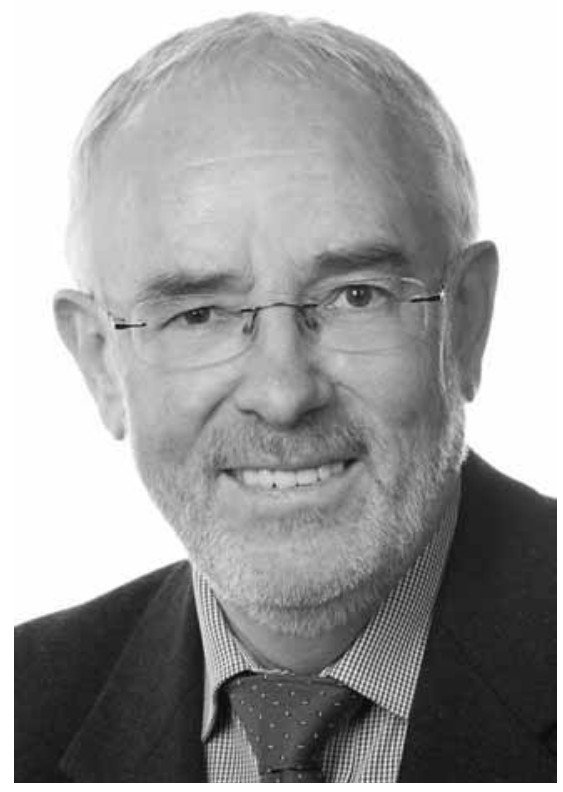

Die Verordnung (EU) 2016/679 des Europäischen Parlaments und des Rates vom 27. April 2016 zum Schutz natürlicher Personen bei der Verarbeitung personenbezogener Daten, zum freien Datenverkehr und zur Aufhebung der Richtlinie 95/46/EG (Datenschutz-Grundverordnung) wird ab dem 25. Mail 2018 in allen Mitgliedstaaten der Europäischen Union gelten und die zentrale Norm für den Datenschutz in den Rechtsordnungen dieser Staaten bilden. Schon heute bestimmt sie die Datenschutzdiskussion und verursacht in allen Mitgliedstaaten Anpassungen der bisher geltenden Datenschutzregelungen und die breite Umstellung von Datenschutzpraktiken.

Die Datenschutz-Grundverordnung verfolgt drei Zielsetzungen: Sie will erstens das Datenschutzrecht unionsweit vereinheitlichen. Durch gleiche wirtschaftliche Bedingungen in der Union will sie zweitens den Binnenmarkt stärken. Schließlich will sie den Datenschutz angesichts der Herausforderungen der technischen Entwicklung modernisieren und den Schutz der Grundrechte verbessern.

Für alle drei Ziele herrschen Zweifel, ob die Verordnung diese erreichen kann. Mehr als 70 Öffnungsklauseln für die Mitgliedstaaten lassen befürchten, dass die unionsweite Vereinheitlichung des Datenschutzrechts noch lange auf sich warten lässt. Die vielen sehr abstrakten Regelungen, die doch unionsweit gelten, müssen von den Mitgliedstaaten umgesetzt werden. Angesichts sehr unterschiedlicher Datenschutzkulturen in den Mitgliedstaaten werden die Interpretationen abstrakter Begriffe, die Abwägungen widerstreitender Interessen und die geforderten Berücksichtigungen vielfältiger Belange in den Mitgliedstaaten stark unterschiedlich ausfallen und gerade nicht zu gleichen wirtschaftlichen Bedingungen in der gesamten Union führen. Ob auch die Modernisierung des Datenschutzrechts, das dritte Ziel der DatenschutzGrundverordnung, verfehlt wird, ist Gegenstand des Schwerpunkts dieses Heftes.

Diese Frage ist nicht so einfach zu beantworten, weil erst einmal geklärt werden muss, welches die künftigen Herausforderungen des Datenschutzrechts sein werden, welche Antworten die Datenschutz-Grundverordnung für sie bietet und wie geeignet diese Antworten für ihre Bewältigung und die Stärkung des Grundrechtsschutzes sind. Die Datenschutz-Grundverordnung ist die Nachfolgeregelung der Datenschutzrichtlinie von 1995. Sie wird bei ihrer Aufhebung zum 25. Mai 2018 beinahe 23 Jahre lang die Datenschutzgrundnorm der Europäischen Union gewesen sein. Wenn die Datenschutz-Grundverordnung einen ähnlichen Geltungszeitraum beansprucht, muss auch sie beanspruchen, für einen vergleichbaren Zeitraum die geeigneten Vorgaben, Strukturen und Instrumente zur Verfügung zu stellen. Daher ist zu fragen, ob sie zumindest für die absehbaren Herausforderungen moderner Informationstechnikanwendungen der kommenden Jahre die passenden Regelungen bietet.

Als Beispiel für bestehende Herausforderungen gelten unter anderem die datengetriebenen Technikanwendungen im Internet, die ihre Dienste geldfrei anbieten, dafür aber sich zusichern lassen, die ihnen zugänglichen personenbezogenen Daten für die Finanzierung ihrer Angebote nutzen zu können. Als Beispiele für bereits erkennbare und demnächst breit sich stellende Herausforderungen aus dem Bereich des Ubiquitous Computing können Smart Health und Smart Home gelten. An ihnen kann erkannt werden, wie neue Anwendungsformen zu einer enormen Zunahme personenbezogener Daten über das alltägliche Leben jedes Einzelnen führen. Alle diese Daten können durch Big Data-Anwendungen ausgewertet und zu äußerst aussagekräftigen Persönlichkeitsprofilen zusammengeführt werden. An diesen Beispielen lässt sich erörtern, wie zukunftsfähig die Datenschutz-Grundverordnung ist. Hierzu sind die absehbaren Herausforderungen, die durch die modernen Technikanwendungen für den Persönlichkeitsschutz und die informationelle Selbstbestim- 
mung verursacht werden, daraufhin zu untersuchen, welche Weiterentwicklungen des Datenschutzrechts sie erfordern. Diese notwendigen datenschutzrechtlichen Innovationen sind mit den neuen Regelungen der Datenschutz-Grundverordnung zu vergleichen.

Diesen Aufgaben stellen sich die Beiträge im Schwerpunkt dieses Heftes. Sie sind aus dem diesjährigen Forum des Competence Center for Applied Security Technology (CAST) in Darmstadt hervorgegangen, das jedes Frühjahr zum Themenkreis „Recht und IT-Sicherheit" veranstaltet wird und in diesem Jahr am 17. März 2016 stattfand. Sie gehen auf Vorträge zurück, die die Autoren dort gehalten und untereinander sowie mit den Teilnehmern des Forums diskutiert haben. Sie wurden für diesen Schwerpunkt unter Berücksichtigung der Anregungen und Erkenntnisse aus den Diskussionen ausgearbeitet. Die Beiträge analysieren und bewerten die Möglichkeiten, mit der ab dem 25. Mai 2018 anzuwendenden Datenschutz-Grundverordnung die absehbaren Herausforderungen des künftigen Datenschutzes zu bewältigen. Sie beschreiben jeweils spezifische Herausforderungen, die bereits heute das Datenschutzrecht vor neue Aufgaben stellen oder dies in naher Zukunft tun werden. Sie analysieren die Regelungen der Datenschutz-Grundverordnung und bewerten die darin zu findenden normativen Lösungen auf ihre Tauglichkeit, die Herausforderungen zu bewältigen. Wo dies nicht der Fall ist, entwickeln sie Vorschläge zu ihrer Fortentwicklung. Der Beitrag von Roßnagel untersucht und kritisiert den grundlegenden Ansatz der Verordnung, die künftigen Herausforderungen gerade mit technik- und risikoneutralen Datenschutzregelungen bewältigen zu wollen. Kugelmann geht der Frage nach, wie mit der Datenschutz-Grundverordnung datengetriebene Geschäftsmodelle datenschutzgerecht gestaltet werden könnten. Jandt und Geminn wählen mit Smart Health und Smart Home jeweils eine Anwendung des Ubiquitous Computing, die unmittelbar vor ihrer breiten Nutzung steht, und analysieren, wie die Datenschutz-Grundverordnung helfen kann, die absehbaren Datenschutzkonflikte zufriedenstellend zu lösen. Das gleiche Untersuchungsziel verfolgt der Beitrag von Richter. Ihn interessiert die Steuerungskraft der Verordnung gegenüber den Herausforderungen verschiedener Anwendungen von Big Data. Schließlich verfolgt Hansen einen etwas anderen Ansatz: Sie fragt nach der Leistungsfähigkeit eines neuen Instruments der Datenschutz-Grundverordnung, nämlich der Datenschutzfolgenabschätzung. Was kann sie dazu beitragen, Datenschutz gegenüber den neuen Herausforderungen zu stärken?

Alle Beiträge kommen - auf unterschiedlichen Wegen und mit unterschiedlicher Gewichtung - letztlich zu dem Ergebnis, dass die Datenschutz-Grundverordnung ihr Ziel einer Modernisierung des Datenschutzrechts und eines verbesserten Grundrechtsschutzes nicht (vollständig) erreicht hat, so dass die Diskussion um eine Modernisierung des Datenschutzrechts - auch nach Inkrafttreten der Datenschutz-Grundverordnung - weitergehen muss.

\section{Alexander Roßnagel}

